\title{
Modeling the Pauli potential in the pair density functional theory
}

\author{
C. Amovilli ${ }^{1}$ and Á. Nagy 2 ,a) \\ ${ }^{1}$ Dipartimento di Chimica e Chimica Industriale, Università di Pisa, Via Risorgimento 35, 56126 Pisa, Italy \\ ${ }^{2}$ Department of Theoretical Physics, University of Debrecen, H-4010 Debrecen, Hungary
}

(Received 29 May 2008; accepted 22 October 2008; published online 25 November 2008)

In the ground state the pair density can be determined by solving a single auxiliary equation of a two-particle problem. A novel method for determining the Pauli potential entering this equation is presented and, starting from a reliable description of the pair density, an analytical expression is derived for atomic systems. Test calculations are presented for $\mathrm{Be}$ and isoelectronic $\mathrm{C}^{2+}$ and $\mathrm{O}^{4+}$ ions. (C) 2008 American Institute of Physics. [DOI: 10.1063/1.3026664]

\section{INTRODUCTION}

Generalized density functional theories have received a growing importance in recent years. For electron systems, the interest has been posed on the pair density as the fundamental variable instead of the one particle density. It turned out that there exist a variational principle for the pair density (analogous to the Hohenberg-Kohn theorems of the density functional theory). It has been shown that-instead of KohnSham equations-in the pair density functional theory ${ }^{1-4}$ the ground state problem of an arbitrary system is reduced to a two-particle problem. The two-particle equation is written ${ }^{1-4}$ as

$$
\begin{array}{r}
{\left[-\frac{1}{2} \nabla_{1}^{2}-\frac{1}{2} \nabla_{2}^{2}+v\left(\mathbf{r}_{1}\right)+v\left(\mathbf{r}_{2}\right)+\frac{N-1}{r}\right.} \\
\left.+v_{P}\left(\mathbf{r}_{1}, \mathbf{r}_{2}\right)\right] \chi\left(\mathbf{r}_{1}, \mathbf{r}_{2}\right)=\mu \chi\left(\mathbf{r}_{1}, \mathbf{r}_{2}\right),
\end{array}
$$

where $v$ is the external potential, $N$ is the number of electrons, and the notation $r=\left|\mathbf{r}_{1}-\mathbf{r}_{2}\right|$ is used. The ground state eigenfunction for this equation, $\widetilde{\chi}_{0}$ say, corresponds to the pair density amplitude and is related to the pair density $n$ of the real system as

$$
n=\frac{N(N-1)}{2}\left|\tilde{\chi}_{0}\right|^{2} \text {. }
$$

Equation (1) contains an unknown term $v_{P}$ of completely kinetic origin. After a density functional analogy $v_{P}$ is called Pauli potential. Equation (1) is analogous to the density functional equation for the square root of the density (which dates back to Thomas and Fermi ${ }^{5}$ and is analyzed by Levy et $a .^{6}{ }^{6}$.

The pair density can be numerically calculated either on the Hartree-Fock (HF) level or on highly correlated level. The pair density can also be determined from Eq. (1) in a rather straightforward way if the Pauli potential is known. However, there are no data for the Pauli potential in the literature, yet. Although recently, the electron-electron cusp condition and asymptotic behavior for the Pauli potential have been derived, ${ }^{7}$ much work is called for to completely

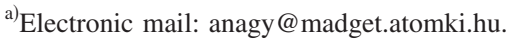

understand how such a potential could be modelized in analogy with the Kohn-Sham potential in ordinary density functional theory. Considering the present knowledge, this kind of work appears extremely difficult. We believe that an important first step, in order to gain more insight in this direction, is the reconstruction of the Pauli potential from a reliable pair density form for some realistic tractable electron system. For these cases, a six variable functional form for $v_{P}$ should be, in principle, obtained. This function, at this point, should be viewed as a source of information expecially with the aim of finding those properties that can be transferred to other systems for which the pair density is unknowm.

Motivated by the above consideration, in this paper we present model Pauli potential for the $\mathrm{Be}$ atom and isoelectronic atomic ions $\mathrm{C}^{2+}$ and $\mathrm{O}^{4+}$. The method, which is intended to capture the main features of $v_{P}$, is based on an ansatz of the form of the pair density amplitude. We generalize the method of Amovilli et al. ${ }^{8}$ The original method was used to obtain the exact Hamiltonian for an analytic ground state wave function for He-like ions. Here, a generalization is presented for producing the Pauli potential from a model pair density amplitude.

The paper is organized as follows. In Sec. II the pair density functional theory is reviewed. In Sec. III a model pair density amplitude and the corresponding potential are presented. Section IV describes numerical examples: the $\mathrm{Be}$ and some isoelectronic atomic ions. The last section is devoted to discussion.

\section{THE PAIR DENSITY FUNCTIONAL THEORY}

First, the pair density functional theory ${ }^{1-3}$ is summarized. Consider the many electron Hamiltonian $H$,

$$
\hat{H}=\hat{T}+\hat{V}_{e e}+\sum_{i=1}^{N} v\left(\mathbf{r}_{i}\right),
$$

where

$$
\hat{T}=\sum_{i=1}^{N}\left(-\frac{1}{2} \nabla_{i}^{2}\right)
$$

is the kinetic energy operator, 


$$
\hat{V}_{e e}=\sum_{i<j}^{N} \frac{1}{\left|\mathbf{r}_{i}-\mathbf{r}_{j}\right|}
$$

is the electron-electron repulsion energy operator, and $v(\mathbf{r})$ is a local external potential. For convenience we consider an even number of particles. The second-ordered reduced density matrix is defined as

$$
\begin{aligned}
n_{2}\left(\mathbf{x}_{1}, \mathbf{x}_{2} ; \mathbf{x}_{1}^{\prime}, \mathbf{x}_{2}^{\prime}\right)= & \frac{N(N-1)}{2} \int \Psi\left(\mathbf{x}_{1}, \mathbf{x}_{2}, \mathbf{x}_{3}, \ldots, \mathbf{x}_{N}\right) \\
& \times \Psi^{*}\left(\mathbf{x}_{1}^{\prime}, \mathbf{x}_{2}^{\prime}, \mathbf{x}_{3}, \ldots, \mathbf{x}_{N}\right) d \mathbf{x}_{3}, \ldots, d \mathbf{x}_{N}
\end{aligned}
$$

where $\mathbf{x}_{i}$ stands for the spatial and the spin coordinates: $\mathbf{r}_{i}, \sigma_{i}$ and the integral symbol when referred to spin denotes summation. The diagonal of the spin-independent secondordered density matrix,

$$
n\left(\mathbf{r}_{1}, \mathbf{r}_{2}\right)=\sum_{\sigma_{1}, \sigma_{2}} n_{2}\left(\mathbf{r}_{1}, \sigma_{1}, \mathbf{r}_{2}, \sigma_{2}\right)
$$

also called pair density is the key quantity.

It is convenient to introduce new position variables

$$
\mathbf{q}_{j}=\left(\mathbf{r}_{j}, \mathbf{r}_{j^{\prime}}\right)=\left(q_{J 1}, q_{J 2}, q_{J 3}, q_{J 4}, q_{J 5}, q_{J 6}\right)
$$

i.e., the pairs will be denoted by capital indices while the particles in each pair will be identified by the corresponding unprimed and primed letters. With the above notation the number of variables is the same as that of the initial system. Each particle is associated with a single pair, i.e., the number of indices $J$ is $N / 2$. The "internal" potential for the particles in pair $J$ is given by

$$
\tilde{v}\left(\mathbf{q}_{j}\right)=\tilde{v}\left(\mathbf{r}_{j}, \mathbf{r}_{j^{\prime}}\right)=\frac{1}{\left|\mathbf{r}_{j}-\mathbf{r}_{j^{\prime}}\right|},
$$

while the interaction between pairs $I$ and $J$ is

$$
\begin{aligned}
W_{I J} & =W\left(\mathbf{q}_{I}, \mathbf{q}_{J}\right)=W\left(\mathbf{r}_{i}, \mathbf{r}_{i^{\prime}} ; \mathbf{r}_{j}, \mathbf{r}_{j^{\prime}}\right) \\
& =\frac{1}{\left|\mathbf{r}_{i}-\mathbf{r}_{j}\right|}+\frac{1}{\left|\mathbf{r}_{i}-\mathbf{r}_{j^{\prime}}\right|}+\frac{1}{\left|\mathbf{r}_{i^{\prime}}-\mathbf{r}_{j}\right|}+\frac{1}{\left|\mathbf{r}_{i^{\prime}}-\mathbf{r}_{j^{\prime}}\right|} .
\end{aligned}
$$

The energy of the pairs due to the external potential is

$$
\hat{U}=\sum_{I=1}^{M} u\left(\mathbf{q}_{J}\right)=\sum_{I=1}^{N}\left(v\left(\mathbf{r}_{j}\right)+v\left(\mathbf{r}_{j^{\prime}}\right)\right)
$$

Defining the operator $\hat{L}$ representing the internal energy of pairs

$$
\hat{L}=\sum_{I=1}^{M}\left(-\frac{1}{2} \nabla_{I}^{2}+\widetilde{v}\left(\mathbf{q}_{I}\right)\right)
$$

the initial Hamiltonian can be expressed as

$$
\hat{H}=\hat{L}+\hat{W}+\hat{U}
$$

where

$$
\hat{W}=\frac{1}{2} \sum_{I \neq J}^{M} W_{I J}
$$

is the interaction energy between different pairs $(M=N / 2)$.

$\hat{H}$ is the same as the initial Hamiltonian, but now it is written in terms of pairs of particles, with $\hat{L}+\hat{U}$ representing the Hamiltonian of independent (noninteracting with each other) pairs and $\hat{W}$ representing the interpair interaction.

The Laplacian in the kinetic energy operator can also be written as

$$
\nabla_{I}^{2}=\nabla_{\mathbf{q}_{I^{\prime}}}^{2}=\nabla_{i}^{2}+\nabla_{i^{\prime}}^{2}=\sum_{\alpha=1}^{6} \frac{\partial^{2}}{\partial q_{I \alpha}^{2}} .
$$

The energy of the independent pairs has the form

$$
Q[n]=\underset{\Psi \rightarrow n}{\min }\langle\Psi|\hat{L}+\hat{W}| \Psi\rangle .
$$

The search of the minimum is over all antisymmetric wave functions $\Psi$ which yield the given $n$. Then the ground state energy can be written as

$$
E=\min _{n}\left\{\frac{1}{N-1} \int u\left(\mathbf{r}_{1}, \mathbf{r}_{2}\right) n\left(\mathbf{r}_{1}, \mathbf{r}_{2}\right) d \mathbf{r}_{1} d \mathbf{r}_{2}+Q[n]\right\} .
$$

The factor $1 /(N-1)$ comes from the normalization of $n$. The density of pair $I$

$$
n\left(\mathbf{q}_{I}\right)=n\left(\mathbf{r}_{i}, \mathbf{r}_{i^{\prime}}\right)=\sum_{\sigma_{i}, \sigma_{i^{\prime}}} n_{2}\left(\mathbf{r}_{i}, \sigma_{i}, \mathbf{r}_{i^{\prime}}, \sigma_{i^{\prime}}\right)
$$

is the pair density in the original space.

The Hohenberg-Kohn theorems ${ }^{9}$ have been generalized for the pair density ${ }^{10,11}$ of the original space. The ground state inequality is

$$
\frac{1}{N-1} \int n(\mathbf{q}) u(\mathbf{q})+Q[n] \geqslant E_{0},
$$

where $E_{0}$ and $n_{0}$ are the ground state energy and the diagonal of the spin-independent second-order density matrix, respectively.

In the pair density functional theory the adiabatic connection is defined by the parametrized Hamiltonian

$$
\hat{H}^{\alpha}=\hat{L}+\alpha \hat{W}+\hat{U}^{\alpha},
$$

where $\hat{U}^{\alpha}=\Sigma_{l} u_{l}^{\alpha}(\mathbf{q})$ is given by the condition that the pair density $n(\mathbf{q})$ of the original space keeps being independent of $\alpha$. For $\alpha=0$ the "noninteracting Hamiltonian"

$$
\hat{H}^{\alpha=0}=\hat{L}+\hat{U}^{\alpha=0}=\sum_{I=1} h_{I}^{\alpha=0}
$$

is obtained. In this auxiliary system the interaction between the pairs is zero and the auxiliary equations have the form

$$
\hat{H}^{0} \Psi^{0}=E^{0} \Psi^{0} .
$$

The wave function in this auxiliary system can be written as a symmetrized expression of antisymmetric two-particle functions $\chi_{1}$ :

$$
\Phi_{0}\left(\mathbf{x}_{1}, \ldots, \mathbf{x}_{N}\right)=\hat{S}\left(\chi_{1}\left(\mathbf{x}_{1}, \mathbf{x}_{2}\right), \ldots, \chi_{M}\left(\mathbf{x}_{N-1}, \mathbf{x}_{N}\right)\right)
$$




$$
\hat{S}=\frac{1}{N !} \sum_{P} \hat{P}
$$

is the symmetrizer operator. $P$ is the permutation operator and the sum is over all permutation of the electron pairs. This wave function is antisymmetric with respect to the exchange of the variables of a single pair and symmetric with respect to the exchange of the pairs. The disadvantage of the present notation is that it does not allow transposition of variables belonging to two different pairs. In the ground state

$$
n(\mathbf{q})=N \frac{N-1}{2} \sum_{\sigma}\left|\chi_{0}\left(\mathbf{x}_{1}, \mathbf{x}_{2}\right)\right|^{2}=N \frac{N-1}{2}\left|\widetilde{\chi}_{0}(\mathbf{q})\right|^{2},
$$

where the two-particle function $\tilde{\chi}_{0}$ satisfies the eigenvalue equation

$$
h^{0}(\mathbf{q}) \widetilde{\chi}_{0}(\mathbf{q})=\left[-\frac{1}{2} \nabla_{\mathbf{q}}^{2}+v_{\text {eff }}(\mathbf{q})\right] \widetilde{\chi}_{0}(\mathbf{q})=\varepsilon_{0} \widetilde{X}_{0}(\mathbf{q}) .
$$

We mention in passing that it is possible to write the pair density in terms of geminals. The present version of the pair density theory has the advantage that the calculation of $n$ is always reduced to the solution of a two-particle equation, that is, the $\mathrm{N}$-body problem can be reduced to a two-body problem. It has been proven ${ }^{1}$ that the auxiliary potential is uniquely determined by the diagonal form of the spinindependent second-order density matrix, and the effective potential is of the form

$$
v_{\text {eff }}(\mathbf{q})=v\left(\mathbf{r}_{1}\right)+v\left(\mathbf{r}_{2}\right)+\frac{N-1}{r_{12}}+v_{p},
$$

where

$$
v_{p}=(N-1) \frac{\delta T_{P}}{\delta n}
$$

and

$$
T_{P}=T-T^{0}
$$

is the difference of the kinetic energies of the real system $(T=\langle\Psi|\hat{T}| \Psi\rangle)$ and the auxiliary system

$$
T^{0}=\sum_{I=1}^{M} \int \chi_{I}^{*}\left(\mathbf{x}_{1}, \mathbf{x}_{2}\right)\left[-\frac{1}{2} \nabla_{\mathbf{q}}^{2}\right] \chi_{I}\left(\mathbf{x}_{1}, \mathbf{x}_{2}\right) .
$$

By a density functional analogy the functional $T_{P}[n]$ is called Pauli energy. The total energy has the form

$$
\begin{aligned}
E[n]= & T^{0}[n]+T_{P}[n]+\int \frac{n(\mathbf{q})}{r_{12}} d \mathbf{q} \\
& +\frac{1}{N-1} \int n(\mathbf{q}) u(\mathbf{q}) d \mathbf{q} .
\end{aligned}
$$

The disadvantage of the present treatment is that it is hard to capture the fermionic structure of an electronic system with a single effective potential. However, we have always a two-particle problem to solve independently of the number of electrons. It is worth making efforts to find adequate approximation for the Pauli potential in order to utilize this benefit. The present study is a step in this direction.
The auxiliary equations can also be derived by constrained search. ${ }^{1,12}$ The two-particle Eq. (26) was later derived $^{13}$ in a different way which is not restricted to even number of electrons.

\section{A MODEL PAIR DENSITY AMPLITUDE AND THE CORRESPONDING POTENTIAL}

As it was shown in the first paper, ${ }^{1}$ the Pauli potential is uniquely determined by the pair density. That is, from the knowledge of $n, v_{P}$ can be given by inverting Eq. (1),

$$
v_{P}\left(\mathbf{r}_{1}, \mathbf{r}_{2}\right)=-K_{\mathrm{loc}}\left(\mathbf{r}_{1}, \mathbf{r}_{2}\right)-w\left(\mathbf{r}_{1}, \mathbf{r}_{2}\right),
$$

where

$$
K_{\mathrm{loc}}\left(\mathbf{r}_{1}, \mathbf{r}_{2}\right)=-\frac{1}{2 \widetilde{\chi}_{0}\left(\mathbf{r}_{1}, \mathbf{r}_{2}\right)}\left[\nabla_{1}^{2}+\nabla_{2}^{2}\right] \widetilde{\chi}_{0}\left(\mathbf{r}_{1}, \mathbf{r}_{2}\right)
$$

and

$$
w\left(\mathbf{r}_{1}, \mathbf{r}_{2}\right)=v\left(\mathbf{r}_{1}\right)+v\left(\mathbf{r}_{2}\right)+\frac{N-1}{r}-\mu .
$$

We have recently proven ${ }^{7}$ that the Pauli potential asymptotically behaves as

$$
v_{P} \rightarrow(N-2)\left(\frac{1}{r_{1}}+\frac{1}{r_{2}}-\frac{1}{r}\right)
$$

when $r_{1} \rightarrow \infty, r_{2} \rightarrow \infty$, and $r \rightarrow \infty$. The electron-electron cusp condition has the form

$$
v_{P}=\frac{2-N}{r}
$$

as $r \rightarrow 0$.

With the aim to reconstruct $v_{P}$ in some functional form, we start out from a model unnormalized pair density amplitude in the form

$$
\chi=\chi^{\mathrm{HF}}\left(\lambda \mathbf{r}_{1}, \lambda \mathbf{r}_{2}\right)(1+g(r)),
$$

where $\chi^{\mathrm{HF}}$ is the HF pair density amplitude and $g(r)$ a reliable correlation function. The variables inside the HF function are properly scaled in accord with a study, ${ }^{14}$ performed on two-electron model atoms, in which it has been shown that the exact ground state wave function for the systems treated can be related to the HF one by a length scaling transformation followed by the insertion of a pair correlation factor. The importance of scaling has been also pointed out in our previous paper on pair density amplitude equation. ${ }^{3}$ Equation (37) above can be used, in principle, for any molecular system. However, in this work, we limit the attention to four-electron atomic ions as a starting point.

In order to be used in some analytical tractable form, the HF pair density amplitude is here expanded in terms of Slater functions, namely,

$$
\chi^{\mathrm{HF}}=\sum_{k l} \Omega_{k l} r_{1}^{p_{k}} r_{2}^{p_{l}} \exp \left(-\alpha_{k} r_{1}-\alpha_{l} r_{2}\right),
$$

where the coefficients $\Omega_{k l}$ and the exponents $\alpha_{k}$ are determined by means of some fitting procedure and the sum is truncated to few terms. 
Now, we follow a recent method ${ }^{8}$ to obtain the potential exactly from the given wave function. From Eqs. (37) and (38) we are led to

$$
\frac{1}{2} \frac{\left(\nabla_{1}^{2}+\nabla_{2}^{2}\right) \chi}{\chi}=V_{1}\left(\mathbf{r}_{1}, \mathbf{r}_{2}\right)+V_{2}\left(\mathbf{r}_{1}, \mathbf{r}_{2}\right)+V_{3}\left(\mathbf{r}_{1}, \mathbf{r}_{2}\right),
$$

$$
\begin{aligned}
& V_{1}\left(\mathbf{r}_{1}, \mathbf{r}_{2}\right)=\nu\left(\mathbf{r}_{1}, \mathbf{r}_{2}\right)+\eta\left(\mathbf{r}_{1}, \mathbf{r}_{2}\right)+\zeta\left(\mathbf{r}_{1}, \mathbf{r}_{2}\right), \\
& \nu\left(\mathbf{r}_{1}, \mathbf{r}_{2}\right)=\frac{\frac{1}{2} \sum_{k l}\left(\bar{\alpha}_{k}^{2}+\bar{\alpha}_{l}^{2}\right) \bar{\Omega}_{k l} r_{1}^{p_{k}} r_{2}^{p_{l}} \exp \left(-\bar{\alpha}_{k} r_{1}-\bar{\alpha}_{l} r_{2}\right)}{\sum_{k l} \bar{\Omega}_{k l} r_{1}^{p_{k}} r_{2}^{p_{l}} \exp \left(-\bar{\alpha}_{k} r_{1}-\bar{\alpha}_{l} r_{2}\right)},
\end{aligned}
$$

where

and

$$
V_{3}\left(\mathbf{r}_{1}, \mathbf{r}_{2}\right)=\frac{2}{r} \frac{d g}{d r} \times \frac{\sum_{k l}\left[\left(\frac{p_{k}}{r_{1}}-\bar{\alpha}_{k}\right)\left(r_{1}-\tilde{\mu} r_{2}\right)+\left(\frac{p_{l}}{r_{2}}-\bar{\alpha}_{l}\right)\left(r_{2}-\tilde{\mu} r_{1}\right)\right] \bar{\Omega}_{k l} r_{1}^{p_{k}} r_{2}^{p_{l}} \exp \left(-\bar{\alpha}_{k} r_{1}-\bar{\alpha}_{l} r_{2}\right)}{(1+g) \Sigma_{k l} \bar{\Omega}_{k l} r_{1}^{p_{k}} r_{2}^{p_{l}} \exp \left(-\bar{\alpha}_{k} r_{1}-\bar{\alpha}_{l} r_{2}\right)} .
$$

$\tilde{\mu}$ denotes the cosine of the angle between $\mathbf{r}_{1}$ and $\mathbf{r}_{2}$,

$$
\tilde{\mu}=\frac{\mathbf{r}_{1} \cdot \mathbf{r}_{2}}{r_{1} r_{2}},
$$

while, accordingly to the length scaling requirements, in Eqs. (41)-(45), we have

$$
\bar{\Omega}_{i j}=\Omega_{i j} \lambda p_{i}+p_{j}
$$

and

$$
\bar{\alpha}_{i}=\alpha_{i} \lambda .
$$

The Schrödinger equation that the model pair density amplitude satisfies is

$$
\begin{aligned}
H \chi\left(\mathbf{r}_{1}, \mathbf{r}_{2}\right) & =\left[-\frac{1}{2} \nabla_{1}^{2}-\frac{1}{2} \nabla_{2}^{2}+V\left(\mathbf{r}_{1}, \mathbf{r}_{2}\right)\right] \chi\left(\mathbf{r}_{1}, \mathbf{r}_{2}\right) \\
& =\mu \chi\left(\mathbf{r}_{1}, \mathbf{r}_{2}\right) .
\end{aligned}
$$

Thus, the potential energy $V\left(\mathbf{r}_{1}, \mathbf{r}_{2}\right)$ in the exact Hamiltonian $H$ has the form

$$
V\left(\mathbf{r}_{1}, \mathbf{r}_{2}\right)=\text { const }+\frac{1}{2} \frac{\left(\nabla_{1}^{2}+\nabla_{2}^{2}\right)}{\chi} .
$$

We can readily see that if we consider the HF, $g=0$ and the potential $V$ reduces to $V_{1}\left(V_{2}=V_{3}=0\right)$. To analyze the terms in the potential $V$ we consider the asymptotic behavior of these terms. We can immediatelly notice that the constant term in Eq. (50) comes from $\nu$, that is, $\mu$ in Eq. (1) is equal to $-\bar{\alpha}_{s}^{2}$, where $\bar{\alpha}_{s}$ is the smallest parameter in the exponent in Eq. (38) and $p_{s}=0$. Terms proportional to $r_{1}^{-1}$, when $r_{1} \rightarrow \infty$, are coming from $\eta$ [Eq. (42)]. That is, $-\bar{\alpha}_{s}\left(p_{s}+1\right) / r_{1}$ should give the corresponding asymptotic limit: $(-Z+N-2) / r_{1}$. Consequently, the relation $\bar{\alpha}_{s}\left(p_{s}-1\right)=Z-N+2$ should hold to insure the correct asymptotic behavior (35). We are led, of course, to the same relation cosidering the limit $r_{2} \rightarrow \infty$. We have to select a correlation function $g$ that the relations (35) and (36) are satisfied as $r \rightarrow \infty$ and $r \rightarrow 0$. With the choice

$$
g(r)=\frac{1}{2} \frac{r}{(1+a r)} \quad(a>0),
$$

the asymptotic relations are fulfilled.

In the next section, we will show a procedure to find reliable values for the parameters $a$ and $\lambda$ for Be isoelectronic atomic ions.

\section{NUMERICAL EXAMPLES: THE BE ATOM AND SOME ISOELECTRONIC ATOMIC IONS}

By way of example, we have performed some calculation of the total energy for $\mathrm{Be}$ atom and isoelectronic atomic ions $\mathrm{C}^{2+}$ and $\mathrm{O}^{4+}$ by using the model pair density amplitude defined in Eq. (37) and the derived Pauli potential. 
TABLE I. Powers and exponents of the Slater-type orbitals used to fit the model pair density amplitude used in this work for $\mathrm{Be}, \mathrm{C}^{2+}$, and $\mathrm{O}^{4+}$.

\begin{tabular}{cccc}
\hline \hline$p_{k}$ & $\alpha_{k}(\mathrm{Be})$ & $\alpha_{k}\left(\mathrm{C}^{2+}\right)$ & $\alpha_{k}\left(\mathrm{O}^{4+}\right)$ \\
\hline 0 & 3.97651 & 5.96857 & 8.06190 \\
0 & 3.00734 & 4.98239 & 7.20878 \\
0 & 1.00912 & 1.49111 & 2.27612 \\
0 & 0.43883 & 0.68890 & 1.47129 \\
1 & 1.02059 & 1.26348 & 2.27198 \\
1 & 0.69171 & 1.01522 & 1.96012 \\
1 & $\ldots$ & $\ldots$ & 1.28699 \\
2 & 1.07922 & 1.45413 & 3.14449 \\
3 & 1.11755 & 1.70673 & $\cdots$ \\
\hline \hline
\end{tabular}

In an earlier paper ${ }^{3}$ we showed that the total energy can be given as

$$
E=E^{0}+T_{p}-\int n\left(\mathbf{r}_{1}, \mathbf{r}_{2}\right) \frac{\delta T_{p}}{\delta n} d \mathbf{r}_{1} d \mathbf{r}_{2},
$$

where

$$
E^{0}=\frac{N}{2} \mu
$$

the Pauli energy $T_{p}$ being defined as $T_{p}=T-T_{w}$, where

$$
\begin{aligned}
T_{w}= & T_{0}=\frac{1}{N-1} \int n^{1 / 2}\left(\mathbf{r}_{1}, \mathbf{r}_{2}\right) \\
& \times\left(-\frac{1}{2} \nabla_{1}^{2}-\frac{1}{2} \nabla_{2}^{2}\right) n^{1 / 2}\left(\mathbf{r}_{1}, \mathbf{r}_{2}\right) d \mathbf{r}_{1} d \mathbf{r}_{2}
\end{aligned}
$$

is a Weizsäcker-type kinetic energy expression. Ayers ${ }^{15}$ has shown that such kind of generalized Weizsäcker-type kinetic energies satisfies a set of bounds in the form

$$
T_{w}^{(1)} \leqslant T_{w}^{(2)} \leqslant \cdots T_{w}^{(N)} \leqslant T_{\mathrm{ex}},
$$

where $T_{\mathrm{ex}}$ is the exact electronic total kinetic energy and the superscript $(j)$ refers to the order of the particle density used to compute $T_{w}$. From the definition of $v_{P}$ in terms of $T_{p}$, namely,

$$
v_{P}=(N-1) \frac{\delta T_{p}}{\delta n}
$$

and from the virial theorem, ${ }^{3}$ the total energy becomes

$$
\begin{aligned}
E= & \frac{1}{N-1} \int n\left(\mathbf{r}_{1}, \mathbf{r}_{2}\right)\left[\mu-v_{P}-\frac{1}{2}\left(\mathbf{r}_{1} \cdot \nabla_{1}\right.\right. \\
& \left.\left.+\mathbf{r}_{2} \cdot \nabla_{2}\right) v_{P}\right] d \mathbf{r}_{1} d \mathbf{r}_{2} .
\end{aligned}
$$

This integral can be evaluated by means of the Monte Carlo method by sampling the two-electron configurations according to the distribution $n$. In this case we can rewrite the energy as the following mean value:

$$
E=\frac{N}{2}\left\langle\mu-v_{P}-\frac{1}{2}\left(\mathbf{r}_{1} \cdot \nabla_{1}+\mathbf{r}_{2} \cdot \nabla_{2}\right) v_{P}\right\rangle .
$$

Subsuming the constant $\mu$ in the costant of the effective potential defined in Eq. (50), the following expression can easily be derived:

$$
\begin{aligned}
E= & \frac{N}{2}\left\langle-V_{L}-\frac{1}{2}\left(\mathbf{r}_{1} \cdot \nabla_{1}+\mathbf{r}_{2} \cdot \nabla_{2}\right) V_{L}\right. \\
& \left.+\frac{1}{2}\left(-\frac{Z}{r_{1}}-\frac{Z}{r_{2}}+\frac{N-1}{r}\right)\right\rangle,
\end{aligned}
$$

which is valid for atoms and where $V_{L}\left(\mathbf{r}_{1}, \mathbf{r}_{2}\right)$ is simply the second term of Eq. (50), namely, that containing the Laplacian of the pair density amplitude. Equation (59) above is the most direct route to get the total energy for an atom within the present pair density functional theory, provided some form of the pair density amplitude is given. We have applied such equation to the isoelectronic systems $\mathrm{Be}, \mathrm{C}^{2+}$, and $\mathrm{O}^{4+}$. We have calculated the HF pair density starting from an even tempered basis set of 30 Gaussian-type orbitals with exponents $\alpha \beta^{k-1}(1 \leqslant k \leqslant 30)$, where $\alpha=0.00015$ and $\beta=2$. This basis set is good enough to approach, for these systems, the HF energy limit within an error of about $10^{-5}$ Hartree. For comparison purposes, together with the energy, we have also evaluated the mean values of some powers of the interelectronic distance $r$. For these moments, and for the systems considered in this work, various data can be found in the literature $^{16-18}$ both for HF and correlated wave functions. Some agreement with these data is requested in order to validate any model pair density amplitude for applications like that presented in this work. The calculation of the aforementioned properties needs the evaluation of the spherically averaged intracule density. The spherically and system averaged pair density is defined as

TABLE II. Total energy $(E)$, Pauli kinetic energy $\left(T_{p}\right)$, and some moments $\left\langle r^{k}\right\rangle$ for Be atom for different choices of the correlation function parameter $a$ and the scaling constant $\lambda$ calculated in this work and comparison with $\mathrm{HF}$ and correlated literature data. Data are in a.u.

\begin{tabular}{lcccccccc}
\hline \hline$a$ & $\lambda$ & $E$ & $T_{p}$ & $\left\langle r^{-2}\right\rangle$ & $\left\langle r^{-1}\right\rangle$ & $\langle r\rangle$ & $\left\langle r^{2}\right\rangle$ & $\left\langle r^{3}\right\rangle$ \\
\hline 4 & 0.9835 & $-14.660(4)$ & $0.878(3)$ & 9.319 & 4.279 & 15.537 & 54.485 & 232.96 \\
5 & 0.9847 & $-14.669(4)$ & $0.864(3)$ & 9.541 & 4.320 & 15.469 & 54.107 & 230.88 \\
6 & 0.9860 & $-14.669(4)$ & $0.855(3)$ & 9.701 & 4.349 & 15.418 & 53.815 & 229.23 \\
HF $^{\mathrm{a}}$ & & -14.573 & 1.005 & 10.536 & 4.489 & 15.120 & 51.956 & 218.11 \\
Corr $^{\mathrm{b}}$ & & -14.667 & $\ldots$ & 9.536 & 4.337 & 15.272 & 52.854 & 222.48 \\
\hline \hline
\end{tabular}

${ }^{\mathrm{a}}$ In the $T_{p}$ column is reported the difference $T_{\mathrm{ex}}-T_{w}^{(1)}$ and moments are from Refs. 16 and 18.

${ }^{b}$ Moments from Ref. 18. 
TABLE III. Total energy (E), Pauli kinetic energy $\left(T_{p}\right)$, and some moments $\left\langle r^{k}\right\rangle$ for $\mathrm{C}^{2+}$ atomic ion for different choices of the correlation function parameter $a$ and the scaling constant $\lambda$ calculated in this work and comparison with HF and correlated literature data. Data are in a.u.

\begin{tabular}{lcccccccc}
\hline \hline$a$ & $\lambda$ & $E$ & $T_{p}$ & $\left\langle r^{-2}\right\rangle$ & $\left\langle r^{-1}\right\rangle$ & $\langle r\rangle$ & $\left\langle r^{2}\right\rangle$ & $\left\langle r^{3}\right\rangle$ \\
\hline 5 & 1.00090 & $-36.538(9)$ & $3.253(8)$ & 25.37 & 7.540 & 8.013 & 14.031 & 29.466 \\
6 & 1.00063 & $-36.538(9)$ & $3.226(8)$ & 25.35 & 7.577 & 7.997 & 13.991 & 29.369 \\
7 & 1.00055 & $-36.539(9)$ & $3.204(8)$ & 25.94 & 7.604 & 7.985 & 13.961 & 29.296 \\
$\mathrm{HF}^{\mathrm{a}}$ & & -36.408 & 3.475 & 27.06 & 7.716 & 7.945 & 13.863 & 29.06 \\
Corr $^{\mathrm{b}}$ & & -36.534 & $\cdots$ & 25.50 & 7.548 & 8.118 & 14.502 & 31.14 \\
\hline \hline
\end{tabular}

${ }^{a}$ In the $T_{p}$ column is reported the difference $T_{\mathrm{ex}}-T_{w}^{(1)}$ and moments are from Refs. 16 and 18.

${ }^{\mathrm{b}}$ Moments from Ref. 16.

$$
f(r)=\int n(\mathbf{r}, \mathbf{R}) \frac{d \Omega_{r}}{4 \pi} d \mathbf{R},
$$

where

$$
\mathbf{R}=\frac{1}{2}\left(\mathbf{r}_{1}+\mathbf{r}_{2}\right) .
$$

This is the spherically averaged intracule density. With our model pair density amplitude this function takes the simple form

$$
f(r)=\mathcal{N}[1+g(r)]^{2} f_{\mathrm{HF}}(\lambda r),
$$

where $f_{\mathrm{HF}}(r)$ is the spherically averaged intracule density from the HF wave function and $\mathcal{N}$ is a normalization constant.

The present approximation is similar to the lowest-order approximation for the short range correlation given by Dal Ri et al. ${ }^{19}$ and rediscussed very recently by Higuchi and Higuchi $^{20}$ in the framework of pair density functional theory. It differs in the precise form of $g(r)$, here taken as defined in Eq. (51). Long range correlation is here in part considered by the renormalization of $\chi$ after the insertion of the factor $(1+g)$ and in part by the length scaling transformation. A more refined treatment with the inclusion of long range correlation will be an objective of future work.

Turning to the three atomic systems studied in this paper, we show in Table I the parameters defining the Slater-type orbitals used to construct the HF pair density amplitude as they result from a fitting of the same accurate function. The complete definition of $\tilde{\chi}_{0}$ depends at this point on the parameter $a$ entering the correlation function $g(r)$ and the scaling factor $\lambda$. We made different choices of such parameters for all the three cases and the final results are collected in Tables II-IV.

The main problem encountered in the calculation of the total energy by Monte Carlo method has been related to the high variance of the function to be averaged which is defined in Eq. (59). This requires a long simulation to achieve an energy mean value with an accuracy of the order of some millihartree. The same occurs for $T_{p}$.

Looking at the results of Tables II-IV, it is evident that the optimal values of the parameters $a$ and $\lambda$ must be found by searching for a compromise between the need of getting reliable values of the moments $\left\langle r^{k}\right\rangle$ and the best energy. The results show also that a considerable fraction of correlation energy has been taken into account. It is also important to notice that the variance becomes larger when the nuclear charge increases but also that our approximation, mainly based on short range correlation, should works better in such cases.

It is also interesting to look at the values of $T_{p}$. From the bounds on the generalized Weizsäcker-type kinetic energy introduced by Ayers ${ }^{15}$ it follows that

$$
0 \leqslant T_{p} \leqslant T_{\mathrm{ex}}-T_{w}^{(1)}
$$

Looking at our results, this inequality is satisfied in the range of $a$ values considered here. Deviations from this behavior must lead to considerations related to $N$-representability.

Finally, it is worthwhile to look at the plots of the effective potential derived by the approximate pair density amplitude. This has been done for the contributions $V_{1}\left(r_{1}, r_{2}\right)$ and $V_{2}(r)$ while $V_{3}\left(r_{1}, r_{2}, r\right)$ cannot be easily shown being depen-

TABLE IV. Total energy $(E)$, Pauli kinetic energy $\left(T_{p}\right)$, and some moments $\left\langle r^{k}\right\rangle$ for $\mathrm{O}^{4+}$ atomic ion for different choices of the correlation function parameter $a$ and the scaling constant $\lambda$ calculated in this work and comparison with HF and correlated literature data. Data are in a.u.

\begin{tabular}{lcccccccc}
\hline \hline$a$ & $\lambda$ & $E$ & $T_{p}$ & $\left\langle r^{-2}\right\rangle$ & $\left\langle r^{-1}\right\rangle$ & $\langle r\rangle$ & $\left\langle r^{2}\right\rangle$ & $\left\langle r^{3}\right\rangle$ \\
\hline 7 & 1.00005 & $-68.40(1)$ & $6.91(1)$ & 49.11 & 10.712 & 5.492 & 6.534 & 9.280 \\
8 & 1.00003 & $-68.40(1)$ & $6.91(1)$ & 49.45 & 10.741 & 5.486 & 6.522 & 9.260 \\
9 & 1.00003 & $-68.41(1)$ & $6.90(1)$ & 49.73 & 10.763 & 5.481 & 6.513 & 9.244 \\
HF $^{\mathrm{a}}$ & & -68.257 & 7.374 & 51.8 & 10.887 & 5.455 & 6.469 & 9.167 \\
Corr $^{\mathrm{b}}$ & & -68.411 & $\ldots$ & 49.17 & 10.694 & 5.570 & 6.769 & 9.843 \\
\hline \hline
\end{tabular}

${ }^{\mathrm{a}}$ In the $T_{p}$ column is reported the difference $T_{\mathrm{ex}}-T_{w}^{(1)}$ and moments are from Refs. 16 and 18.

${ }^{b}$ Moments from Ref. 16. 


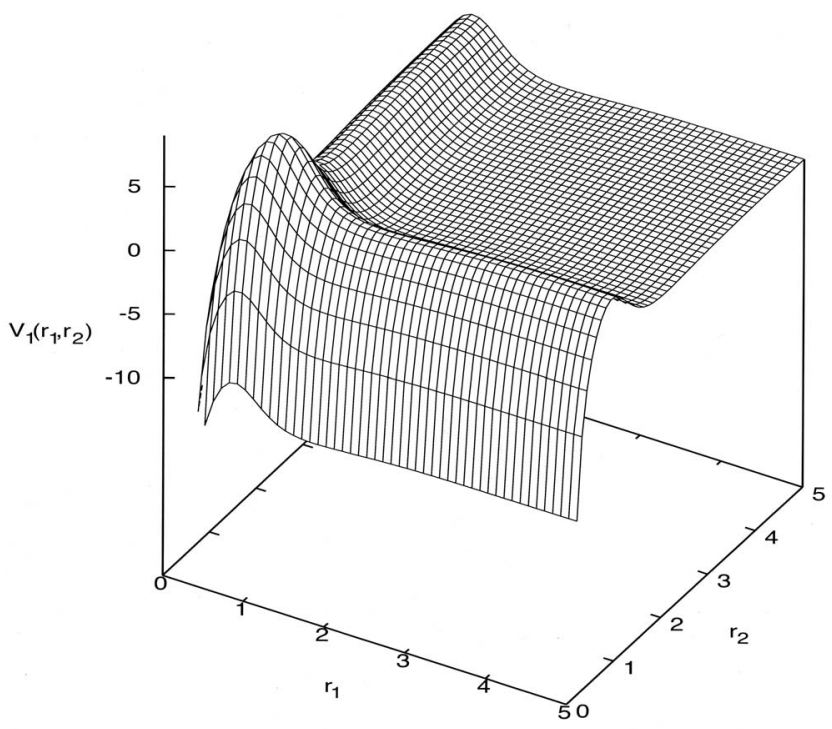

FIG. 1. Plot of the potential energy contribution $V_{1}\left(r_{1}, r_{2}\right)$ for Be. Energies and distances in a.u.

dent on three independent variables. For this purpose, and only for Be, we plot $V_{1}\left(r_{1}, r_{2}\right)$ in Fig. 1 and $V_{2}(r)$ in Fig. 2. From Fig. 1, it is clear that $V_{1}$ is dominated by the external nuclear potential when $r_{1}$ or $r_{2}$ tends to 0 while is about constant for both large $r_{1}$ and $r_{2}$, being $-\mu$ the limit in this case. The ripples of the two dimensional surface of Fig. 1 are instead a consequence of the exchange interaction and determine the shell structure of the one particle density of Be atom. Finally, $V_{2}$, shown in Fig. 2, is always repulsive. For small $r$, it behaves as the electron-electron interaction potential while it goes to zero more rapidly for large $r$. The ripples of $V_{1}$, the long range behavior of $V_{2}$ and the contribution $V_{3}$, are special features of $v_{P}$.

\section{CONCLUSIONS}

In this work, we have illustrated a method to reconstruct the Pauli potential of pair density functional theory for fourelectron atomic ions. The potential is derived by inverting the effective two-electron equation involving the pair density amplitude assuming that the pair density itself can be written in an analytical tractable form. Cusp and asymptotic conditions have been satisfied, and appropriate adjustable parameters have been used in order to reproduce, within a reasonable accuracy, the total energy and some lower moment of the intracule density. Some interesting features of the Pauli potential have been found for the systems treated here. These features are contained in the expressions (40), (44), and (45) for $V_{1}, V_{2}$, and $V_{3} . V_{1}$ and $V_{2}$ include also the nuclear and the electron-electron electrostatic potential energies. Some illustrations are given also in Figs. 1 and 2.

We would like to emphasize that the present method is not restricted to four-electron sytems. In the pair density theory one has to solve an effective two-electron equation independently on the number of electrons. That is, the novel method introduced here to invert the effective two-electron equation can always be applied if the pair density (or the pair density amplitude) is available.

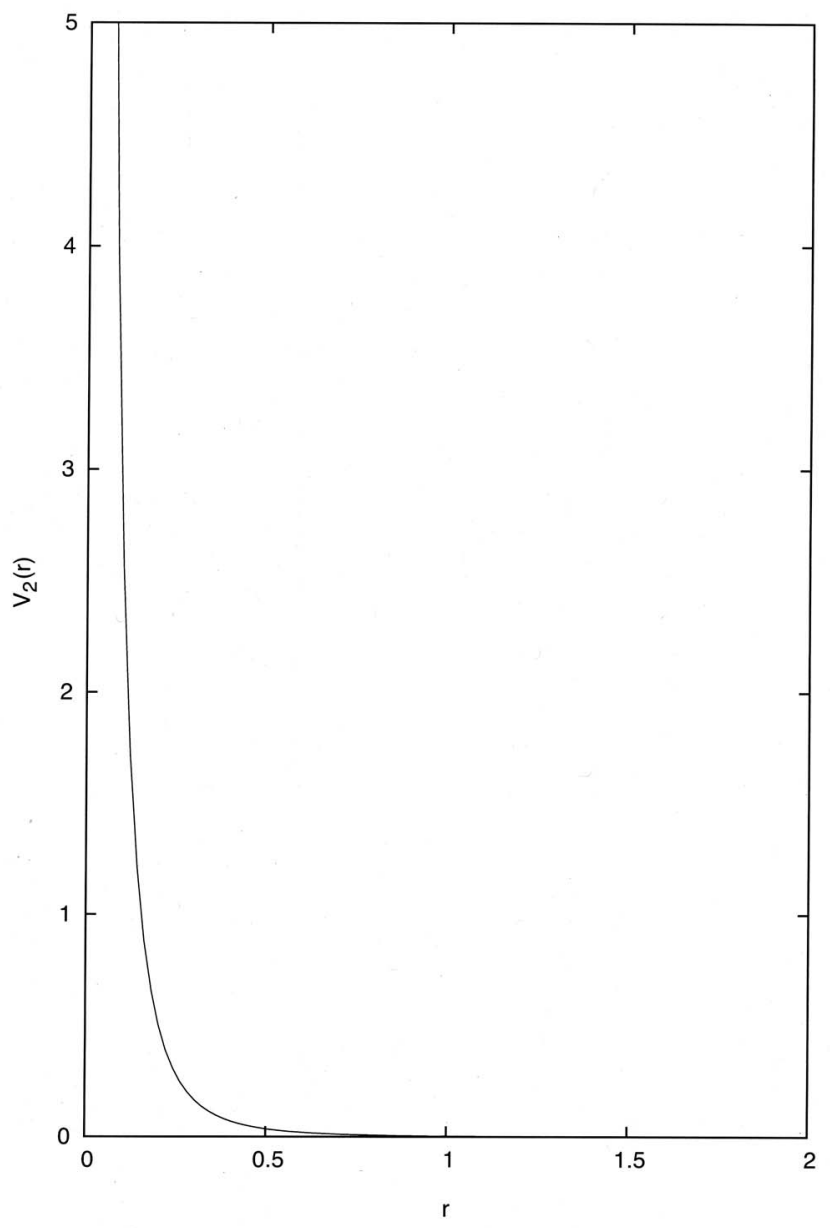

FIG. 2. Plot of the potential energy contribution $V_{2}(r)$ for Be. Energies and distancies in a.u.

For the future, it will be interesting to analyze in details each individual term in order to find a generalization of the above expressions for all polyelectronic systems in a form which does not require the inversion of the effective twoelectron equation worked here.

About $V_{2}$, we would like to refer briefly to the "average pair density theory" of Gori-Giorgi and Savin. In this theory the spherically and system averaged pair density $f(r)$ is determined by simple radial equations conjectured by Gori-Giorgi and Savin, ${ }^{21}$

$$
\left[-\nabla_{r}^{2}+w_{\mathrm{eff}}(r)\right] \phi_{i}(r)=\epsilon_{i} \phi_{i}(r),
$$

the solutions of which give $f(r)$ as

$$
\sum_{i} \theta_{i}\left|\phi_{i}(r)\right|^{2}=f(r),
$$

that is, $f(r)$ is given by a weighted sum of the square of some orthogonal "effective" geminals $\phi_{i}$ with weighting factors of "occupancy" $\theta_{i}$. The potential $w_{\text {eff }}(r)$ in Eq. (64) was approximated as

$$
w_{\mathrm{eff}}(r)=w_{\mathrm{eff}}^{(0)}(r)+w_{\mathrm{eff}}^{c}(r),
$$

where 


$$
w_{\mathrm{eff}}^{(0)}(r)=\frac{\nabla^{2} f_{\mathrm{KS}}^{1 / 2}}{f_{\mathrm{KS}}^{1 / 2}}
$$

and

$$
w_{\mathrm{eff}}^{c}(r)=\left(\frac{1}{r}+\frac{r^{2}}{2 \bar{r}_{s}^{3}}-\frac{3}{2 \bar{r}_{s}}\right) \theta\left(\bar{r}_{s}-r\right) .
$$

$\theta\left(\bar{r}_{s}-r\right)$ is the Heaviside step function and

$$
\bar{r}_{s}=\left(\frac{4 \pi}{3} \bar{\varrho}\right)^{-1 / 3},
$$

where $\bar{\varrho}$ is the average electron density. The correlation potential $w_{\text {eff }}^{c}(r)$, originally proposed by Overhauser, ${ }^{22}$ has been used to solve Eq. (64) for the uniform electron gas. ${ }^{21,23}$ It leads to an accurate description of the short range part of $f$. Our potential $V_{2}\left(\mathbf{r}_{1}, \mathbf{r}_{2}\right)$ (44), using the expression (51) for $g$, has the form

$$
V_{2}\left(\mathbf{r}_{1}, \mathbf{r}_{2}\right)=\frac{1}{r(1+a r)^{2}(1+(a+1 / 2) r)} .
$$

We immediatelly notice that the dominant term in Eq. (70) for small $r$ is $1 / r$. It is the same as the first term in the Overhauser potential, which is also the dominant part of the Overhauser potential for small $r$. Thus the potential $V_{2}\left(\mathbf{r}_{1}, \mathbf{r}_{2}\right)$ has some resemblance to the Overhauser potential. The $1 / r$ term in the Overhauser potential comes from the cusp condition on $f(r) .^{21}$ The dominant term in Eq. (70) for small $r$ has the same origin.

We also mention in passing that it was derived via a double adiabatic connection by one of the present authors ${ }^{4}$ that the square root of the spherically and system averaged pair density is the solution of a simple radial equation, that is, contrary to the theory of Gori-Giorgi and Savin, it is possible to obtain $f(r)$ through a solution of a single equation. If a single geminal is used the fermionic character should be reflected in the potential which is consequently more complicated. If more than one geminals are used the spherically and system averaged pair density has a more complicated form but the potential can be more easily approximated. The number of geminals $N(N-1) / 2$ depends on the number of electrons. Therefore a single geminal approach might gain an important role as the number of electrons increases.

In the density functional theory there has been a growing interest in determining the exact exchange, exchangecorrelation, and Kohn-Sham potentials in the knowledge of the density. Several methods have been worked out. ${ }^{24-29}$ The exact potentials are very useful, for example, to check the accuracy of approximate methods. An analogous problem in the pair density functional theory is to obtain the Pauli potential in the knowledge of the pair density as here the electron-electron iteraction is exactly treated, but the kinetic energy functional is unknown. The problem here is more complicated in the sense that a two-particle potential $v_{P}$ should be calculated, instead of a one-body exchangecorrelation potential of the density functional theory. On the other hand, it is also simpler as only a single equation has to be inverted instead of several Kohn-Sham equations in the density functional theory. The accurate form of the Pauli potential obtained by the present method can be used later to find approximate expressions for it. One has to be, however, extremely careful in the construction because of the $N$-representability problem. ${ }^{10,15,30-43}$

Dal Ri et al. ${ }^{19}$ derived density matrices from Jastrowtype trial wave functions. The pair density used in this work can be considered as the lowest-order approximation to the general $N$-representable pair density presented by $\mathrm{Dal} \mathrm{Ri}$ et al. Consequently, our pair density is, at least approximately, $N$-representable.

\section{ACKNOWLEDGMENTS}

This paper was written in the frame of the Bilateral Scientific Cooperation between Italy and Hungary sponsored by Consiglio Nazionale delle Ricerche and the Hungarian Academy of Sciences in Grant OTKA No. T029469 is gratefully acknowledged.

${ }^{1}$ Á. Nagy, Phys. Rev. A 66, 022505 (2002).

${ }^{2}$ Á. Nagy, in The Fundamentals of Electron Density, Density Matrices and Density Functional Theory in Atoms, Molecules and Solid State, edited by N. I. Gidopoulos and S. Wilson (Kluwer, Dordrecht, 2003) p. 79.

${ }^{3}$ Á. Nagy and C. Amovilli, J. Chem. Phys. 121, 6640 (2004).

${ }^{4}$ Á. Nagy, J. Chem. Phys. 125, 184104 (2006).

${ }^{5}$ L. H. Thomas, Proc. Cambridge Philos. Soc. 23, 542 (1926); E. Fermi, Z. Phys. 48, 73 (1928).

${ }^{6}$ M. Levy, J. P. Perdew, and V. Sahni, Phys. Rev. A 30, 2745 (1984).

${ }^{7}$ Á. Nagy and C. Amovilli, J. Chem. Phys. 128, 114115 (2008).

${ }^{8}$ C. Amovilli, N. H. March, I. A. Horward, and Á. Nagy, Phys. Lett. A 372, 4053 (2008).

${ }^{9}$ P. Hohenberg and W. Kohn, Phys. Rev. 136, B864 (1964).

${ }^{10}$ P. Ziesche, Phys. Lett. A 195, 213 (1994).

${ }^{11}$ A. Gonis, T. C. Schulthess, J. van Ek, and P. E. A. Turchi, Phys. Rev. Lett. 77, 2981 (1996); Phys. Rev. B 56, 9335 (1997).

${ }^{12}$ M. Levy, Proc. Natl. Acad. Sci. U.S.A. 76, 6062 (1979); E. Lieb, Int. J. Quantum Chem. 24, 243 (1983).

${ }^{13}$ F. Furche, Phys. Rev. A 70, 022514 (2004).

${ }^{14}$ C. Amovilli and N. H. March, Chem. Phys. Lett. 378, 167 (2003).

${ }^{15}$ P. W. Ayers, J. Math. Phys. 46, 062107 (2005).

${ }^{16}$ F. J. Gálvez, E. Buendía, and A. Sarsa, J. Chem. Phys. 111, 10903 (1999).

${ }^{17}$ F. J. Gálvez, E. Buendía, and A. Sarsa, Chem. Phys. Lett. 378, 330 (2003).

${ }^{18}$ J. Komasa, W. Cencek, and J. Rychlewski, Phys. Rev. A 52, 4500 (1995).

${ }^{19}$ M. Dal Ri, S. Stringari, and O. Bohigas, Nucl. Phys. A 376, 81 (1982).

${ }^{20}$ M. Higuchi and K. Higuchi, Phys. Rev. A 75, 042510 (2007).

${ }^{21}$ P. Gori-Giorgi and A. Savin, Phys. Rev. A 71, 032513 (2005).

${ }^{22}$ A. W. Overhauser, Can. J. Phys. 73, 683 (1995).

${ }^{23}$ P. Gori-Giorgi and A. Savin, Phys. Rev. A 73, 032506 (2006); Philos. Mag. 86, 2643 (2006); J. Toulouse, P. Gori-Giorgi, and A. Savin, Int. J. Quantum Chem. 106, 2026 (2006).

${ }^{24}$ C. O. Almbladh and A. C. Pedroza, Phys. Rev. A 29, 2322 (1994).

${ }^{25}$ F. Aryasetiawan and M. J. Stott, Phys. Rev. B 38, 2974 (1988); J. Chen and M. J. Stott, Phys. Rev. A 44, 2816 (1991); J. Chen, R. O. Esquivel, and M. J. Stott, Philos. Mag. B 69, 1001 (1994).

${ }^{26}$ Q. Zhao and R. G. Parr, J. Chem. Phys. 98, 543 (1993); R. G. Parr, Philos. Mag. B 69, 737 (1994); Q. Zhao, R. C. Morrison, and R. G. Parr, Phys. Rev. A 50, 2138 (1994).

${ }^{27}$ R. van Leeuwen and E. J. Baerends, Phys. Rev. A 49, 2421 (1994).

${ }^{28}$ Á. Nagy, J. Phys. B 26, 43 (1993); Philos. Mag. B 69, 779 (1994).

${ }^{29}$ A. Görling, Phys. Rev. A 46, 3753 (1992); 51, 4501 (1995).

${ }^{30}$ E. R. Davidson, in Reduced Density Matrices in Quantum Chemistry (Academic, New York, 1976).

${ }^{31}$ P. Ziesche, Int. J. Quantum Chem. 60, 149 (1996).

${ }^{32}$ M. Levy and P. Ziesche, J. Chem. Phys. 115, 9110 (2001).

${ }^{33}$ A. J. Coleman, Rev. Mod. Phys. 35, 668 (1963); A. J. Coleman and V. I. Yukalov, Reduced Density Matrices: Coulson's Challange (SringerVerlag, New York, 2000); J. Cioslowski, Many-Electron Densities and Reduced Density Matrices (Kluwer/Plenum, New York, 2000). 
${ }^{34}$ K. Husimi, Proc. Phys. Math. Soc. Jpn. 22, 264 (1940).

${ }^{35}$ P. O. Löwdin, Phys. Rev. 97, 1474 (1955).

${ }^{36}$ E. R. Davidson, Chem. Phys. Lett. 246, 209 (1995); Phys. Rev. A 1, 30 (1970).

${ }^{37}$ F. Sasaki, Phys. Rev. 138, B1338 (1965).

${ }^{38}$ J. K. Percus, J. Chem. Phys. 122, 234103 (2005); B. Liu and J. K. Percus, Phys. Rev. A 74, 012508 (2006).
${ }^{39}$ M. E. Pistol, Chem. Phys. Lett. 400, 548 (2004); 417, 521 (2006).

${ }^{40}$ P. W. Ayers and E. R. Davidson, Int. J. Quantum Chem. 106, 1487 (2006).

${ }^{41}$ P. W. Ayers, S. Gordon, and M. Levy, J. Chem. Phys. 124, 054101 (2006).

${ }^{42}$ P. W. Ayers and M. Levy, J. Chem. Sci. 117, 507 (2006).

${ }^{43}$ C. Garnod and J. K. Percus, J. Math. Phys. 5, 1756 (1964). 